\title{
How fast can a Peierls-Mott insulator be melted?
}

\author{
C. Sohrt, A. Stange, M. Bauer and K. Rossnagel ${ }^{\star}$
}

Received 17th March 2014, Accepted 6th May 2014

DOI: $10.1039 / \mathrm{c} 4 \mathrm{fd} 00042 \mathrm{k}$

Time- and angle-resolved extreme ultraviolet photoemission spectroscopy is used to directly determine the momentum-dependent electronic structure dynamics in the layered Peierls-Mott insulators $1 T-\mathrm{TaS}_{2}$ and $1 T-\mathrm{TaSe}_{2}$ on the sub-300 fs time scale. Extracted spectroscopic order parameters display a global two-time-scale dynamics indicating a quasi-instantaneous loss of the electronic orders and a subsequent coherent suppression of the lattice distortion on a time scale related to the frequency of the charge-density-wave amplitude mode. After one half-cycle of coherent amplitude-mode vibration, a crossover state between insulator and metal with partially filled-in and partially closed Mott and Peierls gaps is reached. The results are discussed within the wider context of electronic order quenching in complex materials.

\section{Introduction}

Complex materials, in which electron-electron and electron-lattice interactions are strong, exhibit some of the most intriguing phenomena in the quantum world, including (high-temperature) superconductivity, metal-insulator transitions, and ordering phenomena involving charge, orbital, spin, and lattice degrees of freedom. ${ }^{1}$ Most of our knowledge and microscopic understanding of complex materials and their phenomena has been obtained from experimental and theoretical techniques that are applied in or near equilibrium. The nonequilibrium dynamics of complex materials, by contrast, is much less understood, yet powerful techniques and impressive results are emerging. ${ }^{2-13}$

The most important experimental approach to the non-equilibrium regime is by techniques that combine two ultrashort pulses: one for pump excitation of the material and the other as a time-delayed probe. For pumping, near-infrared laser pulses are most commonly used. For probing, photon pulses in the THz to hard $\mathrm{X}$-ray spectral range and electron pulses with energies of a few $10 \mathrm{keV}$ are now almost routinely applied. Thus, many powerful and complementary techniques have been implemented, including time-resolved optical and photoemission spectroscopy ${ }^{\mathbf{1 4 , 1 5}}$ as well as time-resolved X-ray and electron diffraction. ${ }^{\mathbf{1 6}}$ 
With effective temporal resolutions of a few $10 \mathrm{fs}$, these techniques offer a new perspective on complex materials because they can resolve electron and lattice dynamics at or close to the fundamental time scales of electronic processes and ionic motion in solids. The direct dynamical information can be used to temporally dissect complex phases and identify_via temporal discriminationthe dominant degrees of freedom, order parameters, or interactions. ${ }^{17}$ In particular, due to their distinct characteristic time scales, it is almost straightforward to separate electron-electron from electron-phonon interaction effects and coherent from incoherent phonon processes. These capabilities can provide novel insights not only into complex phases but also into thermally accessible phase transitions..$^{3-5,9,12}$ Moreover, pump-probe techniques can be used to investigate whether and to what extent phase transitions can be coherently controlled ${ }^{2,6,11}$ and novel or "hidden" phases can be created that are not thermally accessible.,13

While there are now various experimental techniques available and many different complex materials have been studied, a few central recurrent questions have emerged in the field, namely: how fast and how are symmetry-broken states melted and restored? What is the nature of transient non-equilibrium states? And what can non-equilibrium dynamics teach us about the nature and origin of complex equilibrium phases?

In this work, we address these questions for a paradigmatic example, the layered Peierls-Mott insulators $1 T-\mathrm{TaX}_{2}(\mathrm{X}=\mathrm{S}$ or $\mathrm{Se})$, in which a large-amplitude charge-density wave (CDW) brings on a Mott transition, i.e., electron-phonon and electron-electron interaction are simultaneously strong. ${ }^{18-20}$ The materials serve as a good reference because the ground state is sufficiently complex and generic, rather well understood, and its experimental signatures are strong. $1 T-\mathrm{TaS}_{2}$, in particular, has been extensively studied with time-resolved methods. ${ }^{5,13,17,21-27}$ Yet, the fundamental question about the speed and mechanism of the quenching of the combined Peierls-Mott-state after femtosecond laser excitation has not been investigated in depth.

Here, we use time- and angle-resolved photoemission spectroscopy (trARPES) with extreme ultraviolet (XUV) pulses from high-harmonic generation (HHG) ${ }^{9}$ to track the ultrafast quenching dynamics in $1 T-\mathrm{TaX}_{2}(\mathrm{X}=\mathrm{S}$ or Se). Time-resolved XUV-ARPES is the ideal tool because it combines the high time resolution needed to resolve electron dynamics with the high momentum resolution and coverage needed to probe "spectroscopic order parameters" at different momenta in the electronic structure. Our focus is on the early stages ( $<300 \mathrm{fs}$ ) of the dynamics, i.e., on the suppression rather than the reformation of electronic and lattice order.

Our results reveal a global two-time-scale dynamics of spectral weight and order-parameter transients indicating a quasi-instantaneous loss of Mott and CDW charge order and a subsequent coherent suppression of CDW lattice order on the time scale of the CDW amplitude mode. The resulting transient nonequilibrium state is characterized by filled-in and partially closed gaps at a relaxed periodic lattice distortion. The results are consistent with an explanation of the equilibrium Peierls-Mott state in $1 T-\mathrm{TaX}_{2}(\mathrm{X}=\mathrm{S}$ or Se) in terms of a linear causeand-effect relationship where the Peierls distortion controls the critical parameters of the Mott transition. More generally, the present work provides evidence for a phenomenological hierarchy of the time scales of electronic order-parameter quenching. 


\section{Materials}

$1 T-\mathrm{TaS}_{2}$ and $1 T-\mathrm{TaSe}_{2}$ are isostructural layered compounds consisting of $\mathrm{X}-\mathrm{Ta}-\mathrm{X}$ ( $\mathrm{X}=\mathrm{S}$ and $\mathrm{Se}$ ) sandwiches ${ }^{28}$ Each atomic layer is hexagonally-packed, the coordination around each Ta atom is approximately octahedral, and the interaction between adjacent sandwiches is weak giving rise to highly anisotropic properties. Correspondingly, the electronic structure near the Fermi energy $\left(E_{\mathrm{F}}\right)$ is quasi-twodimensional. In $1 T-\mathrm{TaS}_{2}$, the band structure is made up of completely filled S $3 \mathrm{p}$ valence bands and a partially (nominally $\mathrm{d}^{1}$ ) filled Ta $5 \mathrm{~d}$ band. The Fermi surface has elliptical electron pockets centered on the edges of the hexagonal Brillouin zone [Fig. 1(a)]. ${ }^{19,29}$ The band structure and Fermi surface of $1 T-\mathrm{TaSe}_{2}$ are similar to the ones of $1 T-\mathrm{TaS}_{2}$, except that the hybridization between the Se $4 \mathrm{p}$ and Ta $5 \mathrm{~d}$ orbitals is significantly stronger, possibly even resulting in a Fermi-level crossing of the uppermost Se $4 \mathrm{p}$ valence band..$^{30,31}$

The elliptical Fermi-surface pockets are prone to nesting [arrows in Fig. 1(a)] which in both compounds promotes the formation of an incommensurate CDW (ICCDW) considerably above room temperature. At lower temperatures, the

(a)

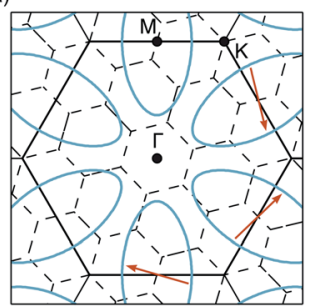

(b)

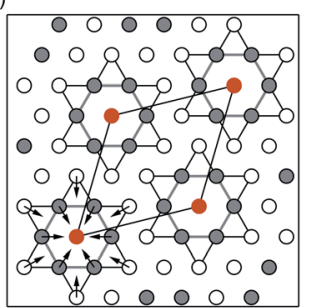

(c)

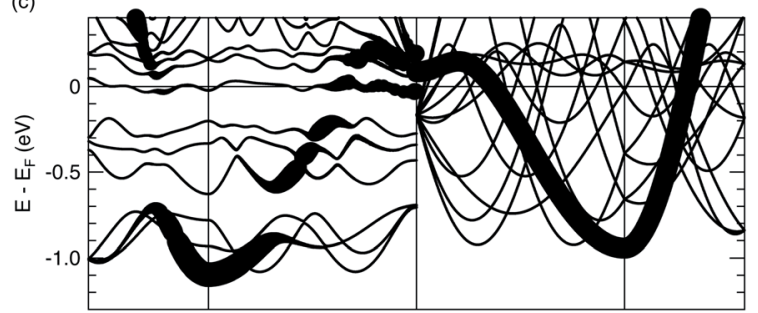

(d)

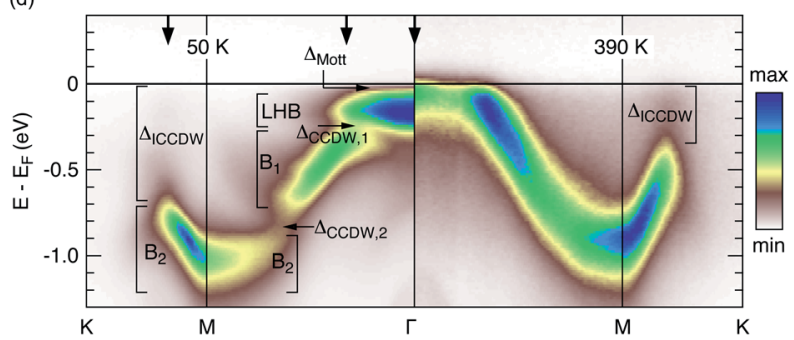

Fig. 1 Characteristic aspects of the lattice and electronic structure of $1 T-\mathrm{TaS}_{2}$ in connection with the commensurate $p(\sqrt{13} \times \sqrt{13}) R 13.9^{\circ}$ charge-density wave. (a) Brillouin zones in the normal (thick solid lines) and reconstructed (thin dashed lines) phases. The unreconstructed Ta $5 d$ Fermi surface has elliptical pockets. Possible nesting vectors are indicated. (b) Unit cell of the periodic lattice distortion (thick lines) and "Star-of-David" clusters (thin lines) in the Ta plane. Arrows indicate the displacements of the Ta atoms from their original positions. (c) Simulated reconstructed band structure (left) and folded-out band structure (right) with Umklapp bands generated by translation through the reciprocal lattice vectors of the reconstructed phase. The spectral weight carried by the electronic states is indicated by the line thickness. (d) Measured ARPES band structure in the commensurate (left) and incommensurate (right) charge-density-wave phase (data recorded with $h \nu=96 \mathrm{eV}$ at the indicated temperatures). Characteristic spectral signatures are labeled. Downward pointing arrows along the top axis denote the momenta at which previous trARPES measurements were performed. ${ }^{17,22,23,27}$ 
ICCDW transforms into a commensurate one, in $1 T-\mathrm{TaS}_{2}$ via an intermediate nearly commensurate CDW phase. The corresponding transition temperatures are still high: $\approx 180-220 \mathrm{~K}$ for $1 T-\mathrm{TaS}_{2}$ and $\approx 470 \mathrm{~K}$ for $1 T-\mathrm{TaSe}_{2} \cdot{ }^{28}$ Intriguingly, the appearance of the commensurate CDW (CCDW) is accompanied by a Mott transition in both compounds. However, transport and ARPES results indicate that only $1 T-\mathrm{TaS}_{2}$ becomes an insulator, whereas $1 T-\mathrm{TaSe}_{2}$ remains metallic. ${ }^{28,31}$ For $1 T-\mathrm{TaSe}_{2}$, it has been argued that the Mott transition is a surface effect. ${ }^{32,33}$

Our focus in this work is on the Peierls-Mott ground state. Fig. 1(b) depicts the characteristic periodic lattice distortion (PLD) in the Ta plane that comes along with the formation of the $p(\sqrt{13} \times \sqrt{13}) R 13.9^{\circ} \mathrm{CCDW}$. The basic motif is a "Starof-David" cluster consisting of a central Ta atom surrounded by two concentric 6Ta-atom rings that are contracted towards the center. ${ }^{34}$ The concomitant modulation of the conduction electron density involves electron transfer from the outer ring towards the inner atoms. The PLD and CDW amplitudes are remarkably large: $\approx 7 \%$ of the in-plane lattice constant ${ }^{34}$ and $\approx 0.5$ electron per Ta atom, ${ }^{35}$ respectively.

Accordingly, the Ta $5 \mathrm{~d}$ electronic structure is strongly reconstructed in the CCDW phase. As shown in Fig. 1(c) for the case of $1 T-\mathrm{TaS}_{2}$, the occupied part of the band structure is split into submanifolds: there are two low-lying three-band submanifolds each filled with six electrons and one distinct and narrow band at $E_{\mathrm{F}}$ hosting the "13th" electron. Since this band has a small width $<100 \mathrm{meV}$ and is nominally half-filled, it is susceptible to a Mott-Hubbard transition. ${ }^{19}$ In the Mott state, the electrons are expected to localize preferentially on the central atom of the Star-of-David cluster. ${ }^{18,19}$ ARPES results have generally confirmed the CCDWinduced reconstruction of the band structure and the opening of the Mott gap predicted by theory. ${ }^{20,36-38}$ Fig. 1(c) and (d) particularly show how well a simple tight-binding model can capture the spectral weight redistribution measured by ARPES. ${ }^{20}$

The static ARPES data of $1 T-\mathrm{TaS}_{2}$ shown in Fig. 1(d) (ref. 17 and 20) set the stage for the time-resolved experiments. There are three different types of spectral gaps that lend themselves as spectroscopic order parameters: the Mott gap $\Delta_{\mathrm{Mott}}$ around $\Gamma$ indicated by the spectral weight suppression between the lower Hubbard band (LHB) and $E_{\mathrm{F}}$, the CCDW gaps $\Delta_{\mathrm{CCDW}, 1}$ and $\Delta_{\mathrm{CCDW}, 2}$ corresponding to the breaks in the band dispersion at about $\frac{1}{3} \overline{\Gamma M}$ and $\frac{2}{3} \overline{\Gamma M}$, and the nesting-induced ICCDW gap $\Delta_{\text {ICCDW }}$ that opens in the vicinity of the $K-M-K$ line and projects into the CCDW phase. ${ }^{20}$ Complementary spectroscopic order parameters are the amounts of "coherent" spectral weight that are transferred from the gapped regions to the three submanifolds denoted LHB, $B_{1}$, and $B_{2}$ in Fig. $1(d)$. We note that momentum resolution and full Brillouin-zone coverage are essential to probe the various spectral signatures.

Previous time-resolved UV- and XUV-ARPES studies on the ultrafast quenching dynamics of the Peierls-Mott state in $1 T-\mathrm{TaS}_{2}$ were performed under moderate to strong excitation, with absorbed pump fluences ranging from 0.135 to $2.2 \mathrm{~mJ} \mathrm{~cm}^{-2} \cdot{ }^{17,22,23,27}$ Spectroscopic order-parameter transients with single rise times were recorded at selected momenta. The focus was mostly on the time constants and not so much on the magnitude of relative intensity changes. Specifically, it was shown that $\Delta_{\mathrm{Mott}}$ and $\Delta_{\mathrm{CCDW}, 1}$ are filled in on an electronic $(<30 \mathrm{fs})$ time scale, while simultaneously the intensity of the LHB is suppressed. ${ }^{17,22,23,27}$ On the other hand, it was shown that a partial closing of 
$\Delta_{\text {ICCDW }}$ and intensity changes in the $\mathrm{B}_{1}$ manifold take place on a vibrational ( $\approx 200 \mathrm{fs}$ ) time scale related to the frequency of the CDW amplitude mode. ${ }^{17,27}$ $1 T-\mathrm{TaSe}_{2}$ has so far not been investigated by trARPES. Yet, the material may be the better suited one because of a larger LHB distance to $E_{\mathrm{F}}\left(\Delta_{\mathrm{Mott}}\right)$ and reduced complexity due to the absence of the nearly commensurate CDW phase.

\section{Experiment}

The trARPES experiments were performed in a pump-probe scheme with an experimental setup sketched in Fig. 2. ${ }^{39}$ The fundamental laser pulses were generated by a Ti:sapphire laser (KMLabs, Griffin) and amplified via a multipass configuration (KMLabs, Dragon) pumped by a Nd:YAG laser (Lee Laser, LDP200MQG). The laser system was operated at a repetition rate of $8.2 \mathrm{kHz}$ and delivered near-infrared (NIR) pulses at a photon energy of $h \nu=1.57 \mathrm{eV}(\lambda=790$ $\mathrm{nm}$ ) with $1 \mathrm{~mJ}$ pulse energy and $32 \mathrm{fs}$ (FWHM) pulse duration. The laser output was split into pump and probe beams using a 10/90 beam splitter.

For photoexcitation of the samples, the fundamental of the laser was used in spolarization. The pump intensity was adjusted using a variable neutral density filter in the pump line. The diameter of the pump beam at the sample was about $300 \mu \mathrm{m}$ (FWHM).

The probe pulses were generated in two steps. The fundamental beam first passed a $200 \mu \mathrm{m}$ thick beta-barium borate (BBO) crystal. The resulting second harmonic ( $h \nu=3.14 \mathrm{eV}, \lambda=395 \mathrm{~nm}$ ) was then focused into an argon-filled commercial high-harmonic-generation (HHG) source (KMLabs, XUUS). ${ }^{40}$ The delivered XUV pulses had a near-Gaussian beam profile, as verified by a beam profile analysis using an image intensifier. A $300 \mathrm{~nm}$ thick aluminum filter was used to block the residual fundamental of the laser transmitted through the HHG source. The XUV radiation was focused with a gold-coated toroidal mirror to a diameter of about $170 \mu \mathrm{m}$ (FWHM) at the sample position, as estimated from the results of ray-tracing simulations. The factor-of-two reduction of spot size compared to the pump beam ensured that a homogeneously excited surface region was probed.

The 14th harmonic $(h \nu=22.1 \mathrm{eV})$ of the fundamental was used for the timeresolved measurements. ${ }^{41}$ The spectral width of the XUV pulses was measured to

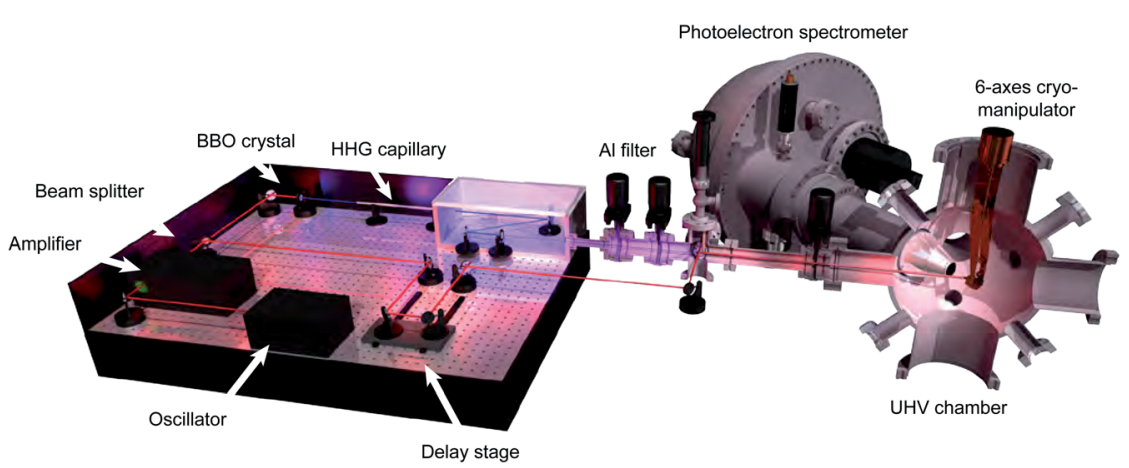

Fig. 2 Schematic illustration of the experimental setup for time-resolved high-harmonicgeneration-based XUV-ARPES. Key components are labeled. For details, see text. 
less than $170 \mathrm{meV}$ employing a grating spectrometer. The temporal width was estimated from NIR-XUV cross-correlation measurements on the conduction-

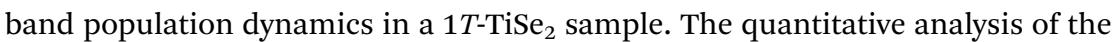
intensity transients yielded an upper limit for the XUV pulse duration of $13 \mathrm{fs}$. The probe pulses were p-polarized.

Photoemission spectra were recorded at a base pressure of $3 \times 10^{-10}$ mbar with a commercial hemispherical electron spectrometer (SPECS, Phoibos 150) equipped with a 2D detection unit for parallel measurement of energy and momentum of the emitted electrons. The total energy resolution achieved in the present study was about $260 \mathrm{meV}$, determined from Fermi-edge spectra of a polycrystalline gold sample. The angular resolution of the experiment was estimated to $1.5^{\circ}$.

$1 T-\mathrm{TaS}_{2}$ and $1 T-\mathrm{TaSe}_{2}$ single crystals were grown from high-purity elements by chemical vapor transport using iodine as transport agent. ${ }^{42}$ Prior to the trARPES experiments, the sample quality was checked by electrical transport as well as high-resolution ARPES and XPS measurements. In the trARPES experiments, the samples were cleaved under ultrahigh vacuum conditions at room temperature using a scotch-tape method. During the trARPES measurements, the equilibrium sample temperature was $100 \mathrm{~K}$.

\section{Results}

The central results of our comparative trARPES study of the Peierls-Mott dynamics in $1 T-\mathrm{TaS}_{2}$ and $1 T-\mathrm{TaSe}_{2}$ are summarized in Fig. 3 and 4 . Fig. 3 displays energy-momentum maps (top row) and energy-distribution curves (bottom row) collected at selected pump-probe delays near the center of the Brillouin zone of
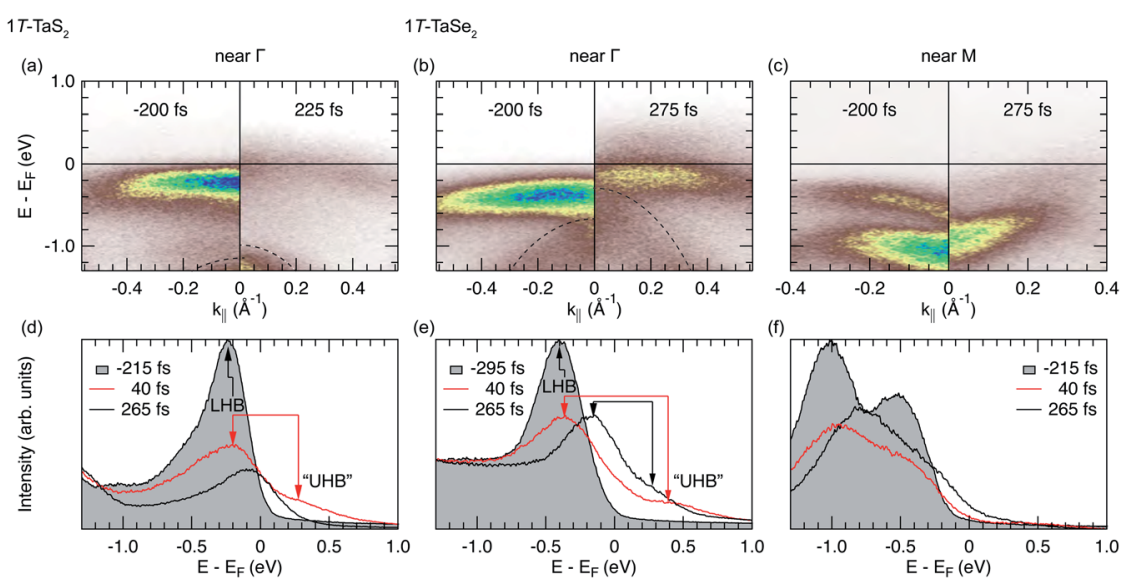

Fig. 3 ARPES snapshots of the electronic structure dynamics in $1 T-\mathrm{TaS}_{2}$ near $\Gamma$ (left column) and in $1 T-\mathrm{TaSe}_{2}$ near $\Gamma$ (middle column) and $M$ (right column) $\left(T=100 \mathrm{~K}, F_{\text {abs }}=\right.$ $3.5 \mathrm{~mJ} \mathrm{~cm}^{-2}, h \nu_{\text {probe }}=22.1 \mathrm{eV}$ ). (a-c) Band maps before and after pumping acquired (a), (b) along the $\Gamma-M$ line and (c) along the $M-K$ line. Dashed lines in (a) and (b) are guides to the eye for the dispersion of the top of the chalcogen valence band. (d-e) Momentumintegrated energy-distribution curves at selected pump-probe delays, as obtained from band maps of the same data sets that the maps in $(a-c)$ were taken from. 
$1 T-\mathrm{TaS}_{2}$

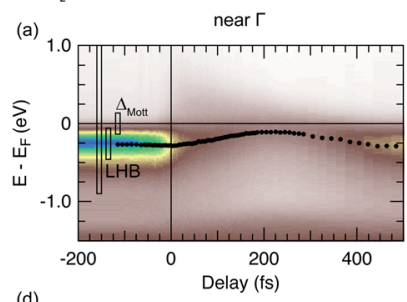

(d)

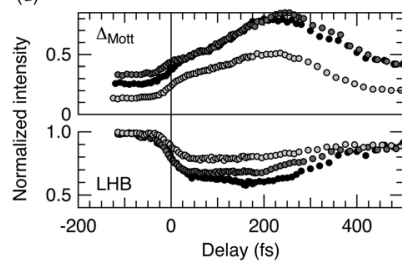

$\circ 1.7 \mathrm{~mJ} / \mathrm{cm}^{2} \circ 3.5 \mathrm{~mJ} / \mathrm{cm}^{2}$
$1 T-\mathrm{TaSe}_{2}$

(b)

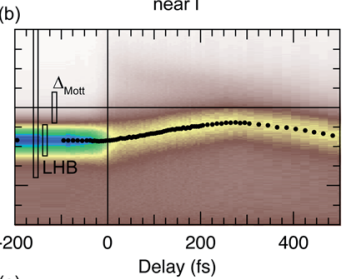

(e)

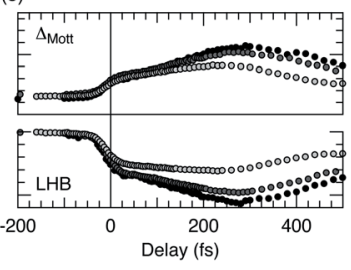

Fig. 4 Time-dependent spectral weight dynamics in $1 T-\mathrm{TaS}_{2}$ near $\Gamma$ (left column) and in $1 T-\mathrm{TaSe}_{2}$ near $\Gamma$ (middle column) and $M$ (right column) $\left(T=100 \mathrm{~K}, h \nu_{\text {probe }}=22.1 \mathrm{eV}\right)$. (a-c) Momentum-integrated energy-distribution curves as a function of pump-probe delay $\left(F_{\mathrm{abs}}=3.5 \mathrm{~mJ} \mathrm{~cm}^{-2}\right)$. Black dots track the time dependence of the center of mass of the spectral weight distribution. (d-e) Photoemission intensity averaged over energy intervals connected with the spectral gaps at $E_{\mathrm{F}}$ (upper panels) and the photoemission peaks (lower panels) $\left(F_{\mathrm{abs}}=1.7-5.2 \mathrm{~mJ} \mathrm{~cm}{ }^{-2}\right)$. For each spectrum, the averaged intensities were divided by the total intensity in the spectral region of the Ta $5 \mathrm{~d}$ band and normalized to the intensity of the corresponding photoemission peak before time zero. The energy integration intervals used are indicated by boxes in $(a-c)$.

$1 T-\mathrm{TaS}_{2}$ (left column) and near the zone center and the zone edge of $1 T-\mathrm{TaSe}_{2}$ (right columns). Fig. 4 has the same column layout as Fig. 3 and shows the detailed time dependence of characteristic energy-distribution curves (top row) and order-parameter transients (bottom row). The electronic structure dynamics of $1 T-\mathrm{TaS}_{2}$ near the Brillouin-zone edge has previously been reported in ref. 17 . It is not shown here because it is almost identical to the one of $1 T-\mathrm{TaSe}_{2}$.

The unpumped energy-momentum maps of $1 T-\mathrm{TaS}_{2}$ and $1 T-\mathrm{TaSe}_{2}$ acquired near $\Gamma$ show the weakly dispersive LHB in excellent agreement with static ARPES results [left panels of Fig. 3(a) and (b), $c f$. Fig. 1(d)]. A notable difference between the two compounds is the larger LHB distance to $E_{\mathrm{F}}$ (by $\approx 200 \mathrm{meV}$ ) in $1 T-\mathrm{TaSe}_{2}$. Another expected difference is the significantly reduced separation between the LHB and the (faintly visible) top of the chalcogen valence band: in $1 T-\mathrm{TaS}_{2}$ there is a clear LHB-S $3 p$ gap, while the LHB and the Se $4 \mathrm{p}$ band in $1 T-\mathrm{TaSe}_{2}$ appear to overlap [dashed lines in Fig. 3(a) and (b)].

The time-selected $\Gamma$-point spectra depicted in Fig. 3(d) and (e) reveal the qualitatively similar dynamics of the LHB in the two compounds. At pump-probe delays $\leq 40 \mathrm{fs}$, the spectral weight carried by the LHB is quasi-instantaneously suppressed and transferred to higher energies; the spectral weight gap at $E_{\mathrm{F}}$ $\left(\Delta_{\mathrm{Mott}}\right)$ is (incoherently) filled in and (coherent) band states above $E_{\mathrm{F}}$ become populated as indicated by a distinct shoulder in the high-energy spectral tail [red spectra in Fig. 3(d) and (e)]. We note that at the rather limited energy resolution of our experiment we find no evidence for the emergence of a mid-gap resonance as reported in ref. 23. 
We tentatively attribute the band states above $E_{\mathrm{F}}$ to the upper Hubbard band (UHB). However, for $1 T-\mathrm{TaS}_{2}$ in equilibrium it is known that the UHB may overlap with other unoccupied Ta $5 \mathrm{~d}$ bands. ${ }^{24}$ The LHB-UHB separation (i.e., the Hubbard $U$ ) is about $510 \mathrm{meV}$ in $1 T-\mathrm{TaS}_{2}$, in agreement with previous trARPES results, ${ }^{23}$ and about $790 \mathrm{meV}$ in $1 T-\mathrm{TaSe}_{2}$, which is about a factor of two larger than the value proposed in a scanning tunneling spectroscopy study. ${ }^{33}$

After the initial quench, on a time scale of $\approx 250$ fs that roughly corresponds to one half-cycle of the CDW amplitude-mode oscillation in these materials, ${ }^{43}$ the suppressed LHB shifts towards $E_{\mathrm{F}}$, thereby (partially) closing the spectral weight gap at $E_{\mathrm{F}}$ [right panels of Fig. 3(a) and (b) and black spectra in Fig. 3(d) and (e)]. Remarkably, in the case of $1 T-\mathrm{TaSe}_{2}$, the signature of the UHB, i.e., the shoulder in the high-energy tail, is still present at the longer time scale. It appears to have shifted to lower energies, suggesting that the LHB-UHB gap has been reduced to about $430 \mathrm{meV}$. However, we note that electron temperature changes at a fixed Hubbard- $U$ may lead to a similar effect. ${ }^{24}$

The unpumped energy-momentum map of $1 T-\mathrm{TaSe}_{2}$ acquired along the Brillouin-zone edge shows the characteristic V-like dispersion of the lowest Ta $5 \mathrm{~d}$ submanifold $\mathrm{B}_{2}$ about the $M$ point. It also shows a weak sign of dispersion away from $E_{\mathrm{F}}$ at the point where the gap opens [left panel of Fig. 3(c), $c f$. Fig. 1(c) and (d)]. The large (nesting-induced) spectral gap $\Delta_{\text {ICCDW }}$ is readily apparent. Inside the gap, a CDW-induced band belonging to the $\mathrm{B}_{1}$ submanifold is observed. This "shadow" band is significantly more intense than the static ARPES data of $1 T-\mathrm{TaS}_{2}$ in Fig. 1(d) would suggest. Possible reasons are electronic structure differences between the two compounds or a matrix-element effect due to the different photon energy used.

Upon photoexcitation, the spectral weight carried by the $\mathrm{B}_{1}$ and $\mathrm{B}_{2}$ bands is quasi-instantaneously suppressed and the previously split bands merge into one spectral feature [red spectrum in Fig. 3(f)]. On the longer time scale, this feature shifts towards $E_{\mathrm{F}}$ and its dispersion straightens out, but a clear spectral gap remains [right panel of Fig. 3(c) and black spectrum in Fig. 3(f)].

Fig. 4 presents the full temporal evolution of the spectral weight dynamics as a function of material and momentum. The data shown in Fig. 4(a)-(c) were obtained from time-resolved energy-momentum maps similar to those depicted in the top row of Fig. 3. The spectra were averaged over momentum intervals of $\approx 0.8 \AA^{-1}$ to improve the statistics of the extracted photoemission intensity transients that are shown in Fig. 4(d)-(f). The photoemission intensity transients represent the temporal evolution of the spectral weight in energy intervals that correspond to the spectral (gap, peak) combinations $\left(\Delta_{\text {Mott }}\right.$, LHB) [Fig. 4 (d) and (e)] and $\left(\Delta_{\text {ICCDW }}, B_{2}\right)$ [Fig. $\left.4(\mathrm{f})\right]$.

The energy-time maps in Fig. 4(a)-(c) vividly illustrate the general, i.e., material- and momentum-independent, two-step dynamics: a quasi-instantaneous suppression of spectral weight is followed by a continuous spectral weight shift towards $E_{\mathrm{F}}$ within 195-280 fs. The fast process occurs on a sub-vibrational (electronic) time scale on the order of the temporal resolution of the experiment; the time constant of the slower component agrees with measured half-cycle times of the CDW amplitude mode (203-224 fs in 1T-TaS 2 (ref. 21-23, 43 and 44) and 222-265 fs in $1 T-\mathrm{TaSe}_{2}$ (ref. 43)), consistent with a displacive excitation of a coherent vibration of that mode. 
All spectroscopic order-parameter transients shown in Fig. 4(d)-(f) also clearly display the two-time-scale dynamics. Initially, spectral weight is promptly transferred from the spectral peaks to the spectral gap regions resulting in the sharp (sub-40 fs) intensity drops and rises, respectively. Subsequently, the intensity changes reflect the shifting of the spectral peaks. The intensity changes become slower and the maximum intensity gains and losses are reached after one halfcycle oscillation of the amplitude mode. When the excitation density is increased, these half-cycle durations become longer, indicating a softening of the CDW amplitude mode. The fast component of the dynamics, on the other hand, remains within the resolution limit.

The peak intensity transients in the lower panels of Fig. 4(d)-(f) additionally display systematic excitation density-dependent amplitude changes. Upon stronger excitation, the amplitude of the amplitude-mode oscillation becomes larger resulting in larger peak shifts out of the fixed energy windows and thus larger spectral weight losses. This behavior is expected and consistent with the fact that the PLD is not completely suppressed, even for the high excitation densities applied here.

The amplitude of the fast, electronic component is more dependent on momentum than on fluence. Near $M$, where only CDW dynamics is probed, the intensity of the $\mathrm{B}_{2}$ peak promptly drops by about $10 \%$, independent of the fluence. By contrast, near $\Gamma$, where mostly Mott dynamics is probed, the initial relative intensity losses of the LHB are larger and wider spread, about 20-35\%. Remarkably, the $20 \%$ intensity quench in $1 T-\mathrm{TaS}_{2}$ at the lowest fluence of $1.7 \mathrm{~mJ} \mathrm{~cm}^{-2}$ agrees with the value reported in ref. 22 and 23 where the absorbed fluence was more than one order of magnitude lower. We attribute both intensity quenches, the one of the LHB near $\Gamma$ and the one of the $\mathrm{B}_{2}$ band near $M$, to the loss of coherent spectral weight resulting from the suppression of charge order of the Mott component and the CDW component, respectively.

Our results thus confirm the general scenario that has emerged from previous trARPES work according to which the charge and lattice orders are suppressed on their own distinct time scales. ${ }^{17,22,23,27}$ However, novel important aspects are added to that scenario here, namely, the global character of the two-time-scale dynamics and the possible change of the Hubbard $U$ in response to the coherent amplitudemode oscillation.

\section{Discussion}

Fig. 5 shows a simple density-of-states scheme that summarizes our trARPES results on the ultrafast electronic structure dynamics in $1 T-\mathrm{TaX}_{2}(\mathrm{X}=\mathrm{S}$ and $\mathrm{Se})$. After femtosecond laser excitation, the strongly reconstructed band structure with clear Peierls and Mott gaps [Fig. 5(a)] is melted on two distinct time scales via two distinct non-equilibrium states. The photoexcited state at $t \leq 40 \mathrm{fs}$ is characterized by filled-in Mott and Peierls gaps and subbands carrying less coherent spectral weight [Fig. 5(b)]. The charge orders associated with Mott localization and CCDW formation are suppressed, whereas the PLD is still frozen. The state is highly non-thermal: neither the electron-phonon system nor the electron system itself will be thermalized. The second state, attained at $t \approx 200 \mathrm{fs}$, also is a crossover state between insulator and metal [Fig. 5(c)]. However, now the gaps are not only filled-in, but they are partially closed. The lattice order had time to relax 
(a)

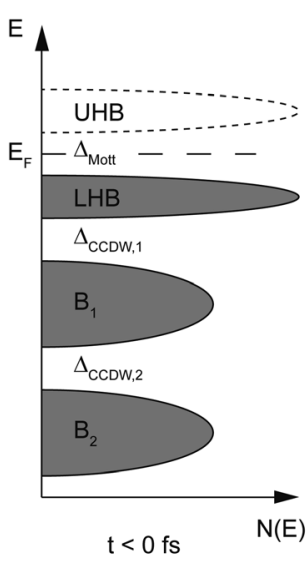

(b)

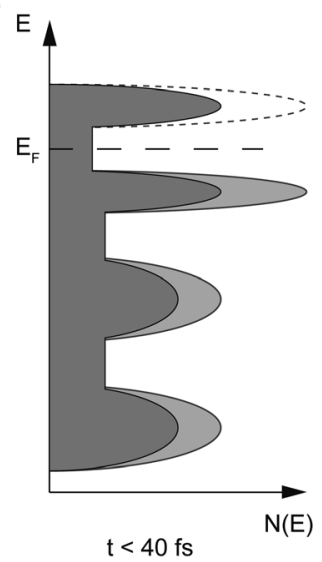

(c)

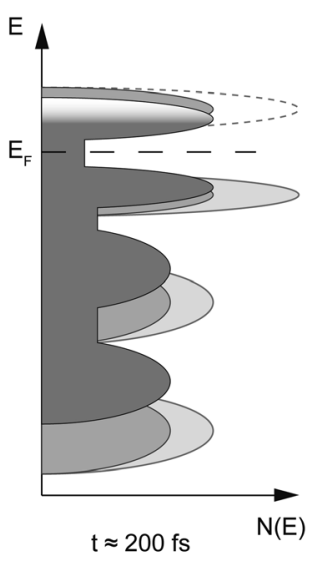

Fig. 5 Schematic representation of the ultrafast electronic structure dynamics in $1 T-\mathrm{TaX}_{2}$ ( $\mathrm{X}=\mathrm{S}$ or $\mathrm{Se}$ ) as seen by trARPES. (a) Peierls-Mott ground state where the density of states below $E_{\mathrm{F}}$ exhibits three distinct peaks separated by $C D W$-induced gaps. The peaks directly below and above $E_{\mathrm{F}}$ are the lower and upper Hubbard band peak, respectively. The gap at $E_{\mathrm{F}}$ is the Mott gap. (b) Non-equilibrium state immediately following photoexcitation. Density-of-states peaks are suppressed and gaps are partially filled in. (c) Non-equilibrium state after about one half-cycle of a coherent amplitude-mode oscillation. Density-ofstates peaks are shifted towards $E_{\mathrm{F}}$ and gaps are partially closed.

coherently so that in addition to the quenched charge orders the PLD will be partially suppressed. The electrons could be expected to have thermalized, but the electron temperature will be vastly higher than the lattice temperature.

These results readily provide answers to the three general questions raised in the introduction. First, regarding the speed limit and mechanism of the PeierlsMott-state melting investigated here, we can conclude that the Peierls-Mott state as a whole-displaying strongly coupled charge and lattice orders-cannot be melted faster than the ions can move coherently. The speed limit for the quenching of this state is thus set by the duration of the relevant lattice vibrational mode. This mode is the CDW amplitude mode. In other words, the bottleneck of the melting process is the coherent breathing motion of the Star-ofDavid clusters [Fig. 1(b)] that is triggered by the femtosecond laser excitation.

Second, as to the nature of the transient non-equilibrium states, we find that from a spectroscopic viewpoint the crossover state at $t \approx 200 \mathrm{fs}$ appears similar to an equilibrium strong-coupling CDW state above the mean-field transition temperature: both states are characterized by smeared, partially closed, but persistent energy gaps ("pseudogaps"). However, the nature of these states is very different. In the non-equilibrium case, we have a hot, disordered electron system decoupled from a coherently fluctuating, but still long-range ordered lattice distortion. The equilibrium state, by contrast, exhibits strongly coupled, but fluctuating short-range electronic and lattice orders. ${ }^{20,45}$

Third, concerning the implications on the equilibrium ground state, we can conclude that the trARPES results are generally consistent with the model described in Sec. II. The Peierls-Mott transition in $1 T-\mathrm{TaX}_{2}(\mathrm{X}=\mathrm{S}$ or Se) is not a chicken-and-egg problem. It is the CCDW distortion that drives the Mott 
transition by controlling the degree of localization on the 13th Ta atom in the center of the Star-of-David cluster. ${ }^{18,19}$ Our results may provide direct evidence for this linear cause-and-effect relationship in that they seem to indicate a reduction of the Hubbard $U$ following the coherent reduction of the PLD amplitude. We note that a vibrational modulation of the Hubbard $U$ has recently also been demonstrated in a time-resolved optical spectroscopy study on an organic Mott insulator. ${ }^{46}$

Finally, we place our results in the wider context of ultrafast photo-induced melting of electronic orders. Fig. 6 presents an overview of measured gap quenching times obtained by time-resolved optical and photoemission spectroscopy from a variety of materials comprising superconductors, CDW systems, and Mott insulators. The data are compiled from ref. 8-10, 17, 27 and 47-51 and plotted as a function of excitation density. The quenching times for $1 T-\mathrm{TaSe}_{2}$ determined in this work are included. The higher sensitivity of time-resolved optical spectroscopy compared to trARPES is immediately seen in the generally lower excitation densities employed in the optical spectroscopy studies.

The plot reveals a phenomenological temporal hierarchy that reflects the distinct characteristic time scales of different processes dominating in the destruction of the three different forms of electronic order.

The quenching times of superconducting states are the slowest, in the range of 0.4-10 ps. This is because the energy transfer from the photo-excited hot carriers to the superconducting condensate is generally inefficient and incoherent. The hot carriers generate a dense population of high-energy (" $2 \Delta$ ") phonons and these phonons subsequently provide the dominant inelastic scattering channel for Cooper-pair breaking. ${ }^{48}$ The quenching time is thus related to an empirical pairbreaking rate.

In CDW systems, as has also been shown in this work, there are two distinct quenching time scales: a slow, vibrational one of about 100-400 fs, and a fast, electronic one on the order of 20-100 fs. The two time scales reflect the fact that a

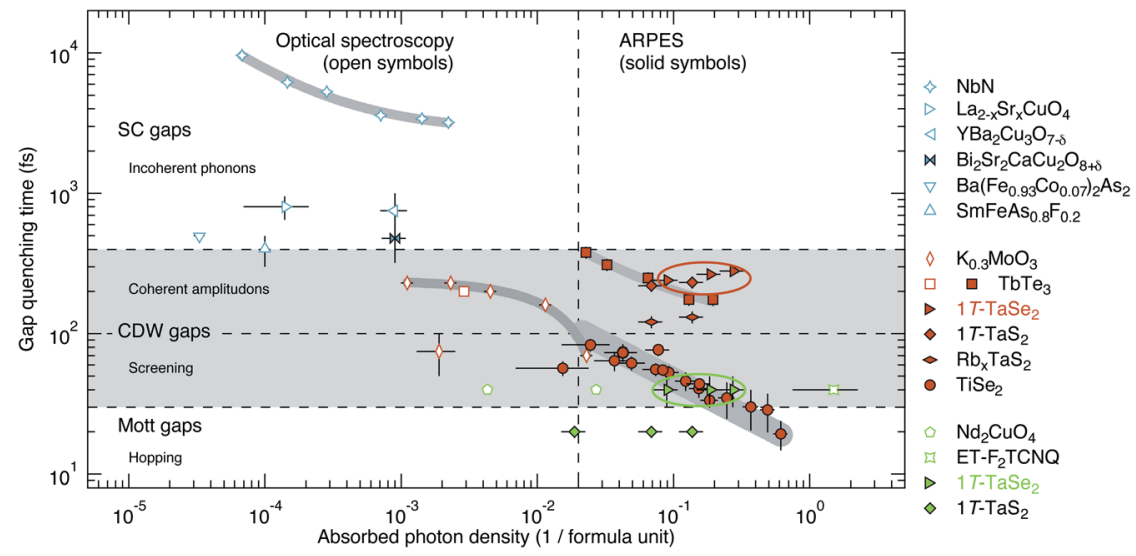

Fig.6 Quenching times of electronic order parameters as a function of excitation density, as measured by time-resolved optical and photoemission spectroscopy for various superconductors, charge-density-wave systems, and Mott insulators. Data compiled from ref. $8-10,17,27$ and 47-51. The results of this work are highlighted by ellipses. 
CDW has two components: a lattice component, the PLD, and an electronic component, the periodic modulation of the conduction-electron density. The relevant collective mode mapping the PLD onto the undistorted lattice is the CDW amplitude mode, and indeed for all systems shown the measured lattice quenching time corresponds well to the half-cycle time of the amplitude mode. Collective electron behavior, on the other hand, is generally related to the plasma frequency. The plasma frequency may therefore be expected to set the time scale on which the electronic component of a CDW is suppressed. ${ }^{9}$ Layered CDW materials of the dichalcogenide family display plasmon energies between 40$140 \mathrm{meV}\left(1 \mathrm{~T}-\mathrm{TiSe}_{2}\right.$ (ref. 52)) and $1.05 \mathrm{eV}\left(2 \mathrm{H}-\mathrm{TaSe}_{2}\right.$ (ref. 53)) which indeed spans a range of screening times of $4-100$ fs. Nonetheless, it is fair to say that the connection between screening and CDW quenching is not yet well established experimentally.

Lastly, Mott-gap quenching appears to be connected with the fastest rise times of about $20-40$ fs. Effectively it will be even faster as typical experimental time resolutions are limited to a few 10 fs. The ultrafast collapse of the Mott gap proceeds via the buildup of coherent or incoherent mid-gap states ${ }^{22-24}$ and the time scale is commonly associated with the fundamental electron hopping rate as given by the bandwidth $W .^{8,17,27}$ However, as suggested in Fig. 5(c), one can also imagine a scenario in which a coherent lattice vibration modulates the parameters $U$ and $W$ such that the system is driven into the metallic regime. The quenching dynamics would then exhibit a slower, vibrational component, similar to the case of a CDW.

Collectively, the data of Fig. 6 corroborate a central motivation for timeresolved studies: the possibility to discriminate fundamental electronic and phononic processes in the time domain. They also show that there is room at the bottom for improved time resolution. The true time scales of the fast electronic processes have not yet been measured.

\section{Conclusion}

In summary, time-resolved NIR pump-XUV probe ARPES has allowed us to study the non-equilibrium dynamics of the isoelectronic Peierls-Mott states of $1 T-\mathrm{TaS}_{2}$ and $1 T-\mathrm{TaSe}_{2}$ in the strong excitation regime. Femtosecond laser excitation in the $\mathrm{mJ} \mathrm{cm}{ }^{-2}$ range suppresses the coupled electron-lattice orders on two distinct time scales. The two charge orders due to Mott localization and the electronic component of the CDW, respectively, are quenched on a resolution-limited electronic time scale, possibly connected with hopping and screening processes. The lattice component of the CDW order, on the other hand, is coherently diminished on a vibrational time scale corresponding to the half-cycle time of the CDW amplitude mode. The Peierls-Mott state is not melted completely, even at the high excitation densities applied. The transient non-equilibrium state is a crossover state between insulator and metal. The Mott and Peierls gaps remain pseudo-gapped; they are filled-in and only partially closed. Overall, the results are consistent with an equilibrium phase-transition mechanism in which the CDW is the driver of the Mott transition. Moreover, the experimentally observed global two-time-scale dynamics fits into a general phenomenological hierarchy of the time scales involved in the quenching dynamics of electronically ordered states. 
So, how fast can a Peierls-Mott insulator be melted? The bottleneck of the photo-induced melting process is the suppression of the PLD and the fastest way to get through this bottleneck is coherent lattice relaxation along the coordinate of the CDW amplitude mode. The period of the amplitude-mode oscillation (or a fraction thereof) thus defines the speed limit of the melting process. The bottleneck time scale for $1 T-\mathrm{TaS}_{2}$ and $1 T$-TaSe $\mathrm{T}_{2}$ directly determined here is $\approx 200-300 \mathrm{fs}$.

Two immediate questions emerge from the presented trARPES results: can the Peierls-Mott state in $1 T-\mathrm{TaS}_{2}$ and $1 T$-TaSe $\mathrm{T}_{2}$ be completely melted non-thermally? And does the (resolution-limited) quenching of the two different electronic orders happen on two different time scales? Both problems provide a challenge for trARPES experiments. The first one requires the use of even higher excitation densities than the ones applied here; the second one calls for sub-10 fs time resolution. In both directions, the price to be paid will be spectral resolutionbecause of pump-induced space-charge broadening and the time-bandwidth product, respectively. Yet, the strong spectroscopic signatures, specifically the large separation of the LHB from $E_{\mathrm{F}}$ in $1 T$-TaSe 2 , may make these experiments feasible.

\section{Acknowledgements}

This work was supported by the DFG via grant BA 2177/9-1 and by the BMBF via grant 05K10FK4.

\section{References}

1 E. Dagotto, Science, 2005, 309, 257.

2 M. Rini, R. Tobey, N. Dean, J. Itatani, Y. Tomioka, Y. Tokura, R. W. Schoenlein and A. Cavalleri, Nature, 2007, 449, 72.

3 F. Schmitt, P. S. Kirchmann, U. Bovensiepen, R. G. Moore, L. Rettig, M. Krenz, J.-H. Chu, N. Ru, L. Perfetti, D. H. Lu, M. Wolf, I. R. Fisher and Z.-X. Shen, Science, 2008, 321, 1649.

4 R. Yusupov, T. Mertelj, V. V. Kabanov, S. Brazovskii, P. Kusar, J.-H. Chu, I. R. Fisher and D. Mihailovic, Nat. Phys., 2010, 6, 681.

5 M. Eichberger, H. Schäfer, M. Krumova, M. Beyer, J. Demsar, H. Berger, G. Moriena, G. Sciaini and R. J. D. Miller, Nature, 2010, 468, 799.

6 D. Fausti, R. I. Tobey, N. Dean, S. Kaiser, A. Dienst, M. C. Hoffmann, S. Pyon, T. Takayama, H. Takagi and A. Cavalleri, Science, 2011, 331, 189.

7 H. Ichikawa, S. Nozawa, T. Sato, A. Tomita, K. Ichiyanagi, M. Chollet, L. Guerin, N. Dean, A. Cavalleri, S. Adachi, T. Arima, H. Sawa, Y. Ogimoto, M. Nakamura, R. Tamaki, K. Miyano and S. Koshihara, Nat. Mater., 2011, 10, 101.

8 S. Wall, D. Brida, S. R. Clark, H. P. Ehrke, D. Jaksch, A. Ardavan, S. Bonora, H. Uemura, Y. Takahashi, T. Hasegawa, H. Okamoto, G. Cerullo and A. Cavalleri, Nat. Phys., 2011, 7, 114.

9 T. Rohwer, S. Hellmann, M. Wiesenmayer, C. Sohrt, A. Stange, B. Slomski, A. Carr, Y. Liu, L. Miaja Avila, M. Kalläne, S. Mathias, L. Kipp, K. Rossnagel and M. Bauer, Nature, 2011, 471, 490.

10 C. L. Smallwood, J. P. Hinton, C. Jozwiak, W. Zhang, J. D. Koralek, H. Eisaki, D.-H. Lee, J. Orenstein and A. Lanzara, Science, 2012, 336, 1137. 
11 K. W. Kim, A. Pashkin, H. Schäfer, M. Beyer, M. Porer, T. Wolf, C. Bernhard, J. Demsar, R. Huber and A. Leitenstorfer, Nat. Mater., 2012, 11, 497.

12 S. de Jong, R. Kukreja, C. Trabant, N. Pontius, C. F. Chang, T. Kachel, M. Beye, F. Sorgenfrei, C. H. Back, B. Bräuer, W. F. Schlotter, J. J. Turner, O. Krupin, M. Doehler, D. Zhu, M. A. Hossain, A. O. Scherz, D. Fausti, F. Novelli, M. Esposito, W. S. Lee, Y. D. Chuang, D. H. Lu, R. G. Moore, M. Yi, M. Trigo, P. Kirchmann, L. Pathey, M. S. Golden, M. Buchholz, P. Metcalf, F. Parmigiani, W. Wurth, A. Föhlisch, C. Schüßler-Langeheine and H. A. Dürr, Nat. Mater., 2013, 12, 882.

13 L. Stojchevska, I. Vaskivskyi, T. Mertelj, P. Kusar, D. Svetin, S. Brazovskii and D. Mihailovic, Science, 2014, 344, 177.

14 D. J. Hilton, R. P. Prasankumar, S. A. Trugman, A. J. Taylor and A. D. Averitt, J. Phys. Soc. Jpn., 2006, 75, 011006.

15 U. Bovensiepen and P. S. Kirchmann, Laser Photonics Rev., 2012, 6, 589.

16 R. J. D. Miller, Science, 2014, 343, 1108.

17 S. Hellmann, T. Rohwer, M. Kalläne, K. Hanff, C. Sohrt, A. Stange, A. Carr, M. M. Murnane, H. C. Kapteyn, L. Kipp, M. Bauer and K. Rossnagel, Nat. Commun., 2012, 3, 1069.

18 P. Fazekas and E. Tosatti, Philos. Mag. B, 1979, 39, 229.

19 K. Rossnagel and N. V. Smith, Phys. Rev. B: Condens. Matter Mater. Phys., 2006, 73, 073106.

20 K. Rossnagel, J. Phys.: Condens. Matter, 2011, 23, 213001.

21 J. Demsar, L. Forró, H. Berger and D. Mihailovic, Phys. Rev. B: Condens. Matter, 2002, 66, 041101(R).

22 L. Perfetti, P. A. Loukakos, M. Lisowski, U. Bovensiepen, H. Berger, S. Biermann, P. S. Cornaglia, A. Georges and M. Wolf, Phys. Rev. Lett., 2006, 97, 067402.

23 L. Perfetti, P. A. Loukakos, M. Lisowski, U. Bovensiepen, M. Wolf, H. Berger, S. Biermann and A. Georges, New J. Phys., 2008, 10, 053019.

24 J. K. Freericks, H. R. Krishnamurthy, Y. Ge, A. Y. Liu and T. Pruschke, Phys. Status Solidi B, 2009, 246, 948.

25 S. Hellmann, M. Beye, C. Sohrt, T. Rohwer, F. Sorgenfrei, H. Redlin, M. Kalläne, M. Marczynski-Bühlow, F. Hennies, M. Bauer, A. Föhlisch, L. Kipp, W. Wurth and K. Rossnagel, Phys. Rev. Lett., 2010, 105, 187401.

26 N. Dean, J. C. Petersen, D. Fausti, R. I. Tobey, S. Kaiser, L. V. Gasparov, H. Berger and A. Cavalleri, Phys. Rev. Lett., 2011, 106, 016401.

27 J. C. Petersen, S. Kaiser, N. Dean, A. Simoncig, H. Y. Liu, A. L. Cavalieri, C. Cacho, I. C. E. Turcu, E. Springate, F. Frassetto, L. Poletto, S. S. Dhesi, H. Berger and A. Cavalleri, Phys. Rev. Lett., 2011, 107, 177402.

28 J. A. Wilson, F. J. Di Salvo and S. Mahajan, Adv. Phys., 1975, 24, 117.

29 M. Bovet, D. Popović, F. Clerc, C. Koitzsch, U. Probst, E. Bucher, H. Berger, D. Naumović and P. Aebi, Phys. Rev. B: Condens. Matter Mater. Phys., 2004, 69, 125117.

30 F. Clerc, M. Bovet, H. Berger, L. Despont, C. Koitzsch, O. Gallus, L. Patthey, M. Shi, J. Krempasky, M. G. Garnier and P. Aebi, J. Phys.: Condens. Matter, 2004, 16, 3271.

31 R. Ang, Y. Miyata, E. Ieki, K. Nakayama, T. Sato, Y. Liu, W. J. Lu, Y. P. Sun and T. Takahashi, Phys. Rev. B: Condens. Matter Mater. Phys., 2013, 88, 115145. 
32 L. Perfetti, A. Georges, S. Florens, S. Biermann, S. Mitrovic, H. Berger, Y. Tomm, H. Höchst and M. Grioni, Phys. Rev. Lett., 2003, 90, 166401.

33 S. Colonna, F. Ronci, A. Cricenti, L. Perfetti, H. Berger and M. Grioni, Phys. Rev. Lett., 2005, 94, 036405.

34 R. Brouwer and F. Jellinek, Phys. B, 1980, 99, 51.

35 M. Eibschutz, Phys. Rev. B: Condens. Matter, 1992, 45, 10914.

36 B. Dardel, M. Grioni, D. Malterre, P. Weibel, Y. Baer and F. Lévy, Phys. Rev. B: Condens. Matter, 1992, 45, 1462(R).

37 L. Perfetti, T. A. Gloor, F. Mila, H. Berger and M. Grioni, Phys. Rev. B: Condens. Matter Mater. Phys., 2005, 71, 153101.

38 R. Ang, Y. Tanaka, E. Ieki, K. Nakayama, T. Sato, L. J. Li, W. J. Lu, Y. P. Sun and T. Takahashi, Phys. Rev. Lett., 2012, 109, 176403.

39 S. Mathias, L. Miaja-Avila, M. M. Murnane, H. Kapteyn, M. Aeschlimann and M. Bauer, Rev. Sci. Instrum., 2007, 78, 083105.

40 A. Rundquist, C. G. Durfee III, Z. Chang, C. Herne, S. Backus, M. M. Murnane and H. C. Kapteyn, Science, 1998, 280, 1412.

41 S. Eich, A. Stange, A. V. Carr, J. Urbancic, T. Popmintchev, M. Wiesenmayer, K. Jansen, A. Ruffing, S. Jakobs, T. Rohwer, S. Hellman, C. Chen, P. Matyba, L. Kipp, K. Rossnagel, M. Bauer, M. M. Murnane, H. C. Kapteyn, S. Mathias and M. Aeschlimann, J. Electron Spectrosc. Relat. Phenom., 2014, DOI: 10.1016/j.elspec.2014.04.013.

42 F. J. Di Salvo, R. G. Maines, J. V. Waszczak and E. Schwall, Solid State Commun., 1974, 14, 497.

43 S. Sugai, Phys. Status Solidi B, 1985, 129, 13.

44 T. Onozaki, Y. Toda, S. Tanda and R. Morita, Jpn. J. Appl. Phys., 2007, 46, 870.

45 W. L. McMillan, Phys. Rev. B: Solid State, 1977, 16, 643.

46 S. Kaiser, S. R. Clark, D. Nicoletti, G. Cotugno, R. I. Tobey, N. Dean, S. Lupi, H. Okamoto, T. Hasegawa, D. Jaksch and A. Cavalleri, Sci. Rep., 2014, 4, 3823.

47 L. Stojchevska, P. Kusar, T. Mertelj, V. V. Kabanov, Y. Toda, X. Yao and D. Mihailovic, Phys. Rev. B: Condens. Matter Mater. Phys., 2011, 84, 180507(R).

48 M. Beck, M. Klammer, S. Lang, P. Leiderer, V. V. Kabanov, G. N. Gol'tsman and J. Demsar, Phys. Rev. Lett., 2011, 107, 177007.

49 A. Tomeljak, H. Schäfer, D. Städter, M. Beyer, K. Biljakovic and J. Demsar, Phys. Rev. Lett., 2009, 102, 066404.

50 F. Schmitt, P. S. Kirchmann, U. Bovensiepen, R. G. Moore, J.-H. Chu, D. H. Lu, L. Rettig, M. Wolf, I. R. Fisher and Z.-X. Shen, New J. Phys., 2011, 13, 063022.

51 H. Okamoto, T. Miyagoe, K. Kobayashi, H. Uemura, H. Nishioka, H. Matsuzaki, A. Sawa and Y. Tokura, Phys. Rev. B: Condens. Matter Mater. Phys., 2011, 83, 125102.

52 G. Li, W. Z. Hu, D. Qian, D. Hsieh, M. Z. Hasan, E. Morosan, R. J. Cava and N. L. Wang, Phys. Rev. Lett., 2007, 99, 027404.

53 A. König, R. Schuster, M. Knupfer, B. Büchner and H. Berger, Phys. Rev. B: Condens. Matter Mater. Phys., 2013, 87, 195119. 